Henry Glover · Dragan Marušič

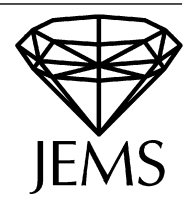

\title{
Hamiltonicity of cubic Cayley graphs
}

Received September 1, 2005 and in revised form September 11, 2006

\begin{abstract}
Following a problem posed by Lovász in 1969, it is believed that every finite connected vertex-transitive graph has a Hamilton path. This is shown here to be true for cubic Cayley graphs arising from finite groups having a $(2, s, 3)$-presentation, that is, for groups $G=\langle a, b| a^{2}=1$, $\left.b^{s}=1,(a b)^{3}=1, \ldots\right\rangle$ generated by an involution $a$ and an element $b$ of order $s \geq 3$ such that their product $a b$ has order 3. More precisely, it is shown that the Cayley graph $X=\operatorname{Cay}\left(G,\left\{a, b, b^{-1}\right\}\right)$ has a Hamilton cycle when $|G|$ (and thus $s$ ) is congruent to 2 modulo 4 , and has a long cycle missing only two adjacent vertices (and thus necessarily a Hamilton path) when $|G|$ is congruent to 0 modulo 4.
\end{abstract}

\section{Introductory remarks}

In 1969, Lovász [22] asked whether every finite connected vertex-transitive graph has a Hamilton path, thus tying together two seemingly unrelated concepts: traversability and symmetry of graphs. (In this article all graphs are assumed to be finite.) The Lovász problem is, somewhat misleadingly, usually referred to as the Lovász conjecture, presumably in view of the fact that, after all these years, a connected vertex-transitive graph without a Hamilton path is yet to be produced. Moreover, only four connected vertex-transitive graphs (having at least three vertices) not possessing a Hamilton cycle are known to exist: the Petersen graph, the Coxeter graph, and the two graphs obtained from them by replacing each vertex with a triangle. All of these are cubic graphs, suggesting perhaps that no attempt to resolve the above problem can bypass a thorough analysis of cubic vertex-transitive graphs. Besides, none of these four graphs is a Cayley graph. This has led to a folklore conjecture that every connected Cayley graph is hamiltonian.

This problem has spurred quite a bit of interest in the mathematical community. In spite of a large number of articles directly and indirectly related to this subject (see [1-9, 12, 16-19, 21, 25, 30, 35,-37| for some of the relevant references), not much progress has been made with regard to either of the two conjectures.

For example, most of the results proved thus far in the case of Cayley graphs depend on various restrictions made either on the class of groups dealt with or on the generating

H. Glover: Department of Mathematics, Ohio State University, Columbus, OH 43210-1174, USA; e-mail: glover@math.ohio-state.edu

D. Marušič: IMFM, University of Ljubljana, Jadranska 19, 1000, Ljubljana, Slovenia, and University of Primorska, Koper, Slovenia; e-mail: dragan.marusic@guest.arnes.si 
sets of Cayley graphs. For example, one may easily see that connected Cayley graphs of abelian groups have a Hamilton cycle. Also, following a series of articles [17, 21, 25] it is now known that every connected Cayley graph of a group with a cyclic commutator subgroup of prime power order is hamiltonian. This result has later been generalized to connected vertex-transitive graphs whose automorphism group contains a transitive subgroup whose commutator subgroup is cyclic of prime power order, with the Petersen graph being the only counterexample [16]. And finally, perhaps the biggest achievement on the subject is a result of Witte (now Morris) which says that a Cayley (di)graph of any $p$-group has a Hamilton cycle [37]. (For further results not explicitly mentioned or referred to here see the survey paper [15].)

In this article we consider hamiltonicity for cubic Cayley graphs arising from groups that have a $(2, s, 3)$-presentation, that is, for groups $G=\langle a, b| a^{2}=1, b^{s}=1$, $\left.(a b)^{3}=1, \ldots\right\rangle$ generated by an involution $a$ and an element $b$ of order $s \geq 3$ such that their product $a b$ has order 3 . More precisely, the following is the main result of this article. (We remark that two vertices $g, h \in G$ in the Cayley graph $\operatorname{Cay}(G, S)$ are adjacent if and only if $g^{-1} h \in S$.)

Theorem 1.1. Let $s \geq 3$ be an integer and let $G=\langle a, b| a^{2}=1, b^{s}=1$, $\left.(a b)^{3}=1, \ldots\right\rangle$ be a group with $a(2, s, 3)$-presentation. Then the Cayley graph $X=$ $\operatorname{Cay}\left(G,\left\{a, b, b^{-1}\right\}\right)$ has a Hamilton cycle when $|G|$ (and thus also $s$ ) is congruent to 2 modulo 4 , and has a cycle of length $|G|-2$ through all but two adjacent vertices, and thus necessarily a Hamilton path, when $|G|$ is congruent to 0 modulo 4 .

Let us comment that the class of groups considered in Theorem 1.1 is by no means restrictive. First, these groups are quotients of the modular group PSL $(2, \mathbb{Z})$. Second, by [23], [24], [33] and [38] every finite nonabelian simple group except the Suzuki groups, a thin family of $\operatorname{PSp}_{n}(q)$ and a thin family of $\operatorname{PSU}_{n}(q)$ groups, $M_{11}, M_{22}, M_{23}, M c L$ and at most finitely many other non-sporadic finite simple groups have a $(2, s, 3)$-presentation. Also, methods similar to those in this article have been used in [13, 14] to find Hamilton cycles in certain Cayley graphs. And third, if $X$ is a cubic arc-transitive graph and $G \leq$ Aut $X$ acts 1-regularly on $X$, then it is easily seen that $G$ has a $(2, s, 3)$-presentation for some $s$. Namely, the ordered pair $(X, G)$ gives rise to a unique orbit of those undirected cycles in $X$ which have the property that each of these cycles is rotated by some automorphism in $G$ (that is, the so called consistent cycles in the terminology of Biggs [11]). These cycles give rise to the faces of the corresponding (orientably) regular map associated with $X$, and their length is then precisely our parameter $s$ in the $(2, s, 3)$-presentation of $G$. Going backwards, the well defined correspondence between these groups (or rather their Cayley graphs) and the class of all those cubic arc-transitive graphs which admit a subgroup acting regularly on the arcs is, geometrically, best seen via the concept of the hexagon graphs, explained in the subsequent section. (However, this correspondence is not 1-1, for a cubic arc-transitive graph may admit nonisomorphic 1-regular groups.)

The article is organized as follows. In Section 2 we describe our method for constructing Hamilton cycles and paths in cubic Cayley graphs of groups having a $(2, s, 3)$ presentation by analyzing six examples of such graphs. They are associated with, respectively, the groups $\mathbb{Z}_{6}$ and $S_{3} \times \mathbb{Z}_{3}$ with a $(2,6,3)$-presentation, the group $S_{4}$ with a 
(2, 4, 3)-presentation, the group $Q_{8} \rtimes S_{3}$ with a $(2,8,3)$-presentation, the group $A_{4}$ with a $(2,3,3)$-presentation and the group $A_{5}$ having a $(2,5,3)$-presentation. In Section 3 we introduce the graph-theoretic concepts of cyclic stability and cyclic connectivity. In particular, we discuss an old theorem of Payan and Sakarovitch [32] which gives the exact cardinality of a maximum cyclically stable set in a cyclically 4-edge-connected cubic graph (Proposition 3.2), a result that proves to be of crucial importance for the purpose of this article. Using a result of Nedela and Škoviera [31] on cyclic connectivity in cubic vertex-transitive graphs (Proposition 3.3), together with an analysis of cubic arc-transitive graphs of girth at most 5 (Proposition 3.4), we then obtain a slight refinement of the above mentioned result of Payan and Sakarovitch (Proposition 3.5), thus laying the groundwork for the proof of Theorem 1.1 which is carried out in Section 4.

\section{The method of proof illustrated}

In this section we give examples illustrating our method of proof of Theorem 1.1. In particular, each Cayley graph we study has a canonical Cayley map given by an embedding of the Cayley graph $X=\operatorname{Cay}\left(G,\left\{a, b, b^{-1}\right\}\right)$ of the $(2, s, 3)$-presentation of a group $G=\left\langle a, b \mid a^{2}=1, b^{s}=1,(a b)^{3}=1, \ldots\right\rangle$ in the corresponding closed orientable surface with the set of faces $F(X)$ comprising $|G| / s$ vertex-disjoint $s$-gons and $|G| / 3$ hexagons. This map is given by using the same rotation of the $b, a, b^{-1}$ edges at every vertex and results in one $s$-gon and two hexagons adjacent to each vertex. Applying the formula for the Euler characteristic

$$
\chi=2-2 g=|V(X)|-|E(X)|+|F(X)|,
$$

we see that the genus $g$ of this surface is $1+(s-6)|G| / 12 s$. As an immediate consequence we have the following observation about $s$ and the order of the group $G$.

Proposition 2.1. Let $s \geq 3$ be an integer and let $G=\langle a, b| a^{2}=1, b^{s}=1,(a b)^{3}$ $=1, \ldots\rangle$ be a group with a $(2, s, 3)$-presentation with order $|G| \equiv 2(\bmod 4)$. Then $s \equiv 2(\bmod 4)$.

In each of the six examples presented here we give a tree of $(|G|-2) / 4$ hexagons if $|G| \equiv 2(\bmod 4)$, and $(|G|-4) / 4$ hexagons if $|G| \equiv 0(\bmod 4)$. This tree of hexagons necessarily contains, respectively, all or all but two of the vertices of the Cayley graph, and as a subspace of the Cayley map is a topological disk. The boundary of that disk is a (simple) cycle passing through, respectively, all or all but two adjacent vertices of the Cayley graph. We give two examples in the case $|G| \equiv 2(\bmod 4)$ and four examples in the case $|G| \equiv 0(\bmod 4)$. In each example we show the tree of hexagons in the Cayley map, in the first case giving rise to a Hamilton cycle of the graph and in the second case giving rise to a long cycle missing only two adjacent vertices. Finally, we do show a Hamilton cycle in the Cayley graph in the case $|G| \equiv 0(\bmod 4)$ when the tree of $(|G|-4) / 4$ hexagons does not give a Hamilton cycle in the Cayley graph. We do this by exhibiting a Hamilton tree of faces in the Cayley map (a tree of faces such that each 
vertex of the Cayley graph lies in the boundary of at least one of these faces) by using an appropriate number of $s$-gons. In Examples 2.2 and 2.3 we have $|G| \equiv 2(\bmod 4)$ and $s \equiv 2(\bmod 4)$, in Examples 2.4 and 2.5 we have $|G| \equiv 0(\bmod 4)$ and $s \equiv 0(\bmod 4)$, and in Examples 2.6 and 2.7 we have $s \equiv 1(\bmod 2)$ and thus $|G| \equiv 0(\bmod 4)$.

Note that the above construction has a direct translation into a more graph-theoretic language by associating with the Cayley graph $X=\operatorname{Cay}\left(G,\left\{a, b, b^{-1}\right\}\right)$ of $G$ the so called hexagon graph $\operatorname{Hex}(X)$ whose vertex set consists of all the hexagons in $X$ arising from the relation $(a b)^{3}$, with two hexagons adjacent in $\operatorname{Hex}(X)$ if they share an edge in $X$. It may be easily seen that $\operatorname{Hex}(X)$ is nothing else than the so called orbital graph of the left action of $G$ on the set $\mathcal{H}$ of left cosets of the subgroup $H=\langle a b\rangle$, arising from the suborbit $\{a H, a b a H, a b a b a H\}$ of length 3. (But note that $a H=b H$ and so $a b a H=a b^{2} H$ and $a b a b a H=b^{-1} H$.) More precisely, the graph has vertex set $\mathcal{H}$, with adjacency defined as follows: an arbitrary coset $x H$ is adjacent to precisely the three cosets $x b H, x b^{-1} H$ and $x a b^{2} H$. Clearly, $G$ acts 1-regularly on $\operatorname{Hex}(X)$. Conversely, let $X$ be a cubic arc-transitive graph $Y$ admitting a 1-regular action of a subgroup $G$ of Aut $Y$. Let $v \in V(Y)$ and let $h$ be a generator of $H=G_{v} \cong \mathbb{Z}_{3}$. Then there must exist an element $a \in G$ such that $G=\langle a, h\rangle$ and such that $Y$ is isomorphic to the orbital graph of $G$ relative to the suborbit $\left\{a H, h a H, h^{2} a H\right\}$. Moreover, a short computation shows that $a$ may be chosen to be an involution, and letting $b=a h$ we get the desired presentation for $G$. There is therefore a well defined correspondence between these two classes of objects, as noted in the introductory section. However, this correspondence is not 1-1, for a cubic arc-transitive graph may possess nonisomorphic 1-regular subgroups. A typical example is the Möbius-Kantor graph on 16 vertices, that is, the generalized Petersen graph $G P(8,3)$, which admits two nonisomorphic 1-regular subgroups, one with a $(2,8,3)$-presentation and the other with a $(2,12,3)$-presentation. The former and the corresponding Cayley graph is discussed in Example 2.5 below. (Note that the generalized Petersen graph $G P(n, r)$, where $n \geq 3$ and $1 \leq r \leq\lfloor n / 2\rfloor$, has vertices $u_{i}, v_{i}, i \in \mathbb{Z}_{n}$, and edges of the form $u_{i} v_{i}, u_{i} u_{i+1}, v_{i} v_{i+r}, i \in \mathbb{Z}_{n}$.)

The trees of hexagonal faces in the associated Cayley map of $X$ (mentioned in the first paragraph) then correspond to vertex subsets in $\operatorname{Hex}(X)$ inducing trees with the property that the complement in $V(X)$ either is an independent set when $|G| \equiv 2(\bmod 4)$, or induces a subgraph with a single edge when $|G| \equiv 0(\bmod 4)$. That this approach works in general will follow from the results given in Section 3 .

Example 2.2. In the middle picture of Figure 1 we show a trivial tree of hexagons (consisting of a single hexagon) whose boundary is a Hamilton cycle in the toroidal Cayley
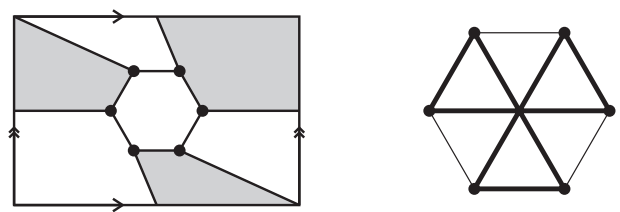

Fig. 1. A (trivial) Hamilton tree of faces in a toroidal Cayley map of $K_{3,3}$ giving rise to a Hamilton cycle, and the associated hexagon graph. 
map of $X=K_{3,3}$, the Cayley graph of the group $G=\mathbb{Z}_{6}$ with a (2,6,3)-presentation $\left\langle a, b \mid a^{2}=b^{6}=(a b)^{3}=1, \ldots\right\rangle$, where $a=3$ and $b=1$. The left picture shows the corresponding hexagon graph $\Theta_{2}$, and the right picture shows the corresponding Hamilton cycle in $X$.

Example 2.3. In the middle picture of Figure 2 we show a Hamilton tree of hexagons whose boundary is a Hamilton cycle in the toroidal Cayley map of the Pappus graph $X$, a Cayley graph of the group $G=S_{3} \times \mathbb{Z}_{3}$ with a $(2,6,3)$-presentation $\langle a, b| a^{2}=$ $\left.b^{6}=(a b)^{3}=1, \ldots\right\rangle$, where $a=((12), 0)$ and $b=((13), 1)$. The left picture shows this same tree in the corresponding hexagon graph $K_{3,3}$, and the right picture shows the corresponding Hamilton cycle in $X$.
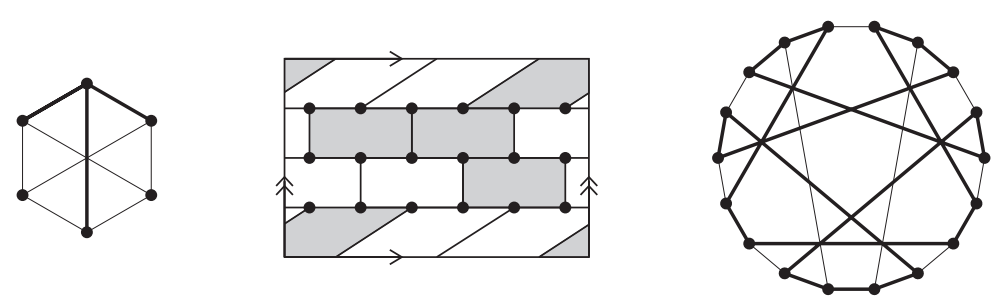

Fig. 2. A Hamilton tree of faces in a toroidal Cayley map of the Pappus graph giving rise to a Hamilton cycle, and the associated hexagon graph.

Example 2.4. In the middle picture of Figure 3 we show a tree of hexagons whose boundary is a cycle missing only two adjacent vertices in the spherical Cayley map of a Cayley graph $X$ of the group $G=S_{4}$ with a $(2,4,3)$-presentation $\left\langle a, b \mid a^{2}=b^{4}=(a b)^{3}=1\right\rangle$, where $a=(12)$ and $b=(1234)$. The left picture shows this same tree in the corresponding hexagon graph $Q_{3}$, the cube, and the right picture shows a modified tree of faces, including also a square, whose boundary is a Hamilton cycle in this map.
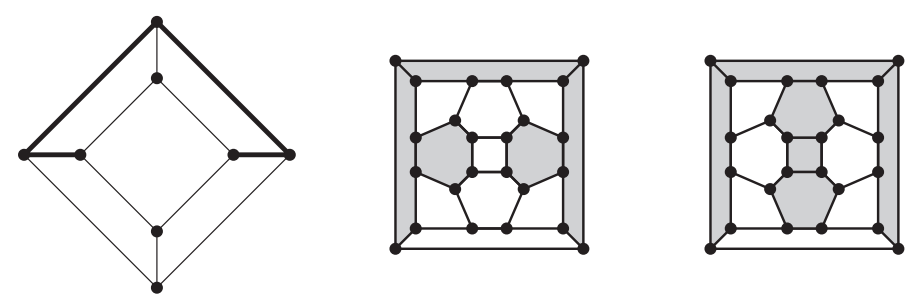

Fig. 3. A tree of faces in the spherical Cayley map of a Cayley graph of $S_{4}$ giving rise to a cycle missing two adjacent vertices, the associated hexagon graph, and a modified Hamilton tree of faces.

Example 2.5. In the middle picture of Figure 4 we give the genus 2 Cayley map of a Cayley graph $X$ of the group $G=Q_{8} \rtimes S_{3}$ with a $(2,8,3)$-presentation $\langle a, b| a^{2}=b^{8}=$ $\left.(a b)^{3}=1, \ldots\right\rangle$, where $a=(1,(23))$ and $b=(i,(12))$. The action of the transposition (12) $\in S_{3}$ on $Q_{8}$ is given by the rule: $i(12)=-j, j(12)=-i, k(12)=-k$, and the rules of action of the other two transpositions are then obvious. In particular, $i(123)=$ $i(23)(12)=j$, and similarly $j(123)=k$ and $k(123)=i$. It is then easily checked 

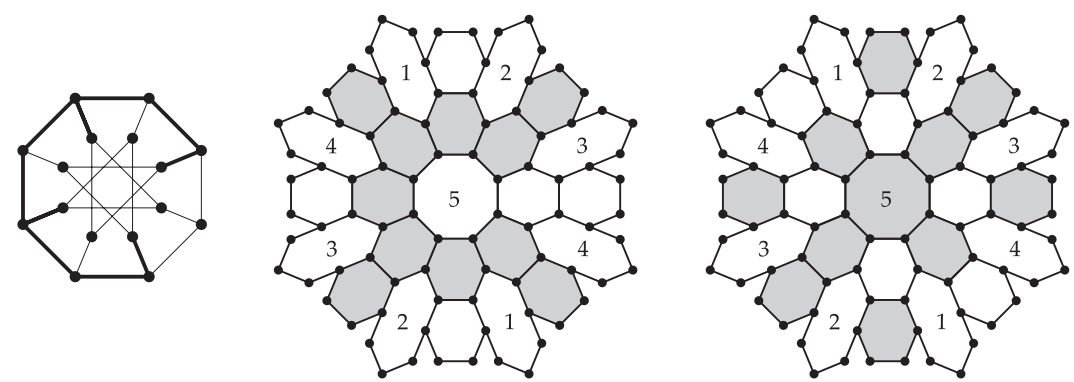

Fig. 4. A tree of faces in the genus 2 Cayley map of a Cayley graph of $Q_{8} \rtimes S_{3}$ giving rise to a cycle missing two adjacent vertices, the associated hexagon graph, and a modified Hamilton tree of faces.

that $a$ is an involution, $b$ has order 8 and $a b$ has order 3. Note that this map is given by identifying antipodal octagons as numbered (and the associated adjacency of hexagons). Note also that the sixth octagon is omitted from this picture, but occurs as the outer edges of the outer hexagons. We show a tree of hexagons in this map, whose boundary is a cycle missing only two adjacent vertices. The left picture shows this same tree in the corresponding hexagon graph, the Möbius-Kantor graph of order 16, and the right picture shows a Hamilton tree of faces, including also an octagon, whose boundary is a Hamilton cycle in this map.

Example 2.6. In the middle picture of Figure 5 we show a tree of hexagons whose boundary is a cycle missing only two adjacent vertices in the spherical Cayley map of a Cayley graph $X$ of the group $G=A_{4}$ with a $(2,3,3)$-presentation $\left\langle a, b \mid a^{2}=b^{3}=(a b)^{3}=1\right\rangle$, where $a=(12)(34)$ and $b=(123)$. The left picture shows this same tree in the corresponding hexagon graph $K_{4}$, and the right picture shows a Hamilton tree of faces, including also two triangles, whose boundary is a Hamilton cycle in this map.
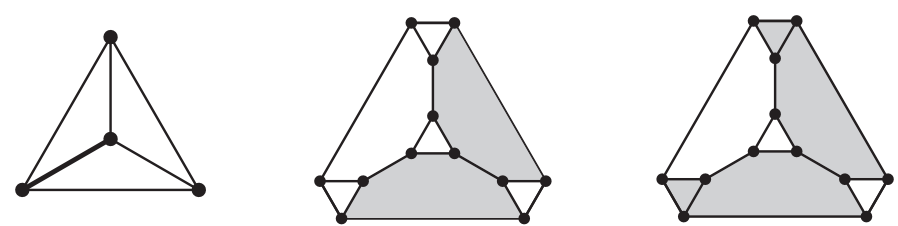

Fig. 5. A tree of faces in the spherical Cayley map of a Cayley graph of $A_{4}$ giving rise to a cycle missing two adjacent vertices, the associated hexagon graph, and a modified Hamilton tree of faces.

Example 2.7. In the middle picture of Figure 6 we show a tree of hexagons whose boundary is a cycle missing only two adjacent vertices in the spherical Cayley map of a Cayley graph $X$ of the group $G=A_{5}$ with a $(2,5,3)$-presentation $\left\langle a, b \mid a^{2}=b^{5}=(a b)^{3}=1\right\rangle$, where $a=(12)(34)$ and $b=(12345)$. The left picture shows this same tree in the corresponding hexagon graph, the dodecahedron, and the right picture shows a Hamilton tree of faces, including also two pentagons, whose boundary is a Hamilton cycle in this map. 

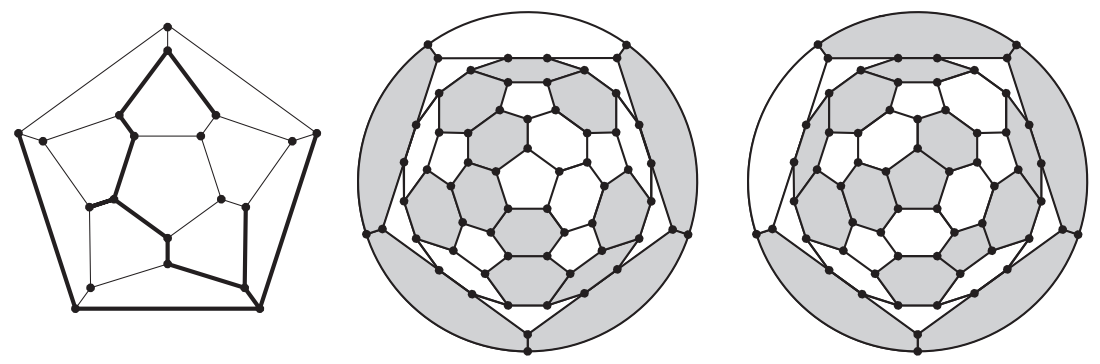

Fig. 6. A tree of faces in the spherical Cayley map of a Cayley graph of $A_{5}$ giving rise to a cycle missing two adjacent vertices, the associated hexagon graph, and a modified Hamilton tree of faces.

\section{Cyclic stability and cyclic connectivity}

A successful application of the method described in the previous section depends heavily on two purely graph-theoretic results. The first one, due to Payan and Sakarovitch [32], goes back to 1975 and deals with maximum cardinalities of vertex subsets in cubic graphs inducing acyclic subgraphs, whereas the second one, due to Nedela and Škoviera [31], is somewhat more recent and concerns cyclic connectivity of vertex-transitive graphs.

Following [32], a paper that is presumably not readily available, we say that, given a graph (or more generally a loopless multigraph) $X$, a subset $S$ of $V(X)$ is cyclically stable if the induced subgraph $X[S]$ is acyclic (a forest). The cardinality $|S|$ of a maximum cyclically stable subset $S$ of $V(X)$ is said to be the cyclic stability number of $X$. The following result giving an upper bound on the cyclic stability number is due to Jaeger [20]. For the sake of completeness we include its proof.

Proposition 3.1 (Jaeger, 1974). Let $X$ be a cubic loopless multigraph of order $n$ and let $S$ be a maximum cyclically stable subset of $V(X)$. Then

$$
|S|=(3 n-2 c-2 e) / 4,
$$

where $c$ is the number of connected components (trees) in $X[S]$ and $e$ is the number of edges in $X[V(X) \backslash S]$. In particular, $|S| \leq(3 n-2) / 4$.

Proof. Let $V=V(X)$. First, in view of maximality of $S$, a vertex in $V \backslash S$ has at most one neighbor in $V \backslash S$, so that each of the $e$ edges in $X[V \backslash S]$ is an isolated edge. Now let $f$ and $g$ denote, respectively, the number of edges in $X[S]$ and the number of edges with one endvertex in $S$ and the other in $V \backslash S$. Then $f=|S|-c$ and $g=|S|+2 c$. Of course, $e+f+g=3 n / 2$ and so $e+2|S|+c=3 n / 2$, giving us the desired expression for $|S|$. Now, clearly, the maximum value for $|S|$ occurs when $e=0$, that is, when $V \backslash S$ is an independent set of vertices, and when at the same time $c=1$, that is, when $X[S]$ is a tree.

In order to explain the result of Payan and Sakarovitch, we need to introduce the concept of cyclic connectivity. Let $X$ be a connected graph. A subset $F \subseteq E(X)$ of edges of $X$ is said to be cycle-separating if $X-F$ is disconnected and at least two of its components 
contain cycles. We say that $X$ is cyclically $k$-edge-connected if no set of fewer than $k$ edges is cycle-separating in $X$. Furthermore, the edge cyclic connectivity $\zeta(X)$ of $X$ is the largest integer $k$ not exceeding the Betti number $|E(X)|-|V(X)|+1$ of $X$ for which $X$ is cyclically $k$-edge-connected. (This distinction is indeed necessary as, for example, the theta graph $\Theta_{2}, K_{4}$ and $K_{3,3}$ possess no cycle-separating sets of edges and are thus cyclically $k$-edge-connected for all $k$, but their edge cyclic connectivities are 2, 3 and 4, respectively.)

In [32, Théorème 5], Payan and Sakarovitch proved that in a cyclically 4-edge-connected cubic graph the above upper bound for its cyclic stability number given in Proposition 3.1 is always attained. More precisely, bearing in mind the expression for the cyclic stability number given in formula (1) of Proposition 3.1. the following result may be deduced from [32, Théorème 5].

Proposition 3.2 (Payan, Sakarovitch, 1975). Let X be a cyclically 4-edge-connected cubic graph of order $n$, and let $S$ be a maximum cyclically stable subset of $V(X)$. Then $|S|=\lfloor(3 n-2) / 4\rfloor$ and more precisely, the following hold.

(i) If $n \equiv 2(\bmod 4)$ then $|S|=(3 n-2) / 4$, and $X[S]$ is a tree and $V(X) \backslash S$ is an independent set of vertices.

(ii) If $n \equiv 0(\bmod 4)$ then $|S|=(3 n-4) / 4$, and either $X[S]$ is a tree and $V(X) \backslash S$ induces a graph with a single edge, or $X[S]$ has two components and $V(X) \backslash S$ is an independent set of vertices.

The connection between cyclic stability and hamiltonicity is now becoming more transparent. Let $G$ be a group with a $(2, s, 3)$-presentation, $X$ be the corresponding Cayley graph and $Y=\operatorname{Hex}(X)$ be its hexagon graph. As described in the previous section, it is precisely the fact that one is able to decompose the vertex set $V(Y)$ into two subsets, the first one inducing a tree, and its complement being an independent set of vertices, that enabled us to produce a Hamilton cycle in the original graph $X$ for the (2, 6, 3)-presentations of $\mathbb{Z}_{6}$ and $S_{3} \times \mathbb{Z}_{3}$. Further, with a slight modification, when the decomposition is such that the first set induces a tree and its complement induces a subgraph with a single edge, then a long cycle missing only two adjacent vertices is produced in $X$. Therefore if $|G|$, and hence the order of the hexagon graph $\operatorname{Hex}(X)$, is congruent to 2 modulo 4, then part (i) of Proposition 3.2 does the trick, provided of course that $\operatorname{Hex}(X)$ is indeed a cyclically 4-edge-connected graph. On the other hand, if $|G|$, and hence the order of $\operatorname{Hex}(X)$, is divisible by 4 , then we are not quite there yet, for only one of the possibilities given in part (ii) of Proposition 3.2 will allow us to construct a long cycle in the original graph $X$. In what follows we explore this situation by, first, bringing into the picture an important result on edge cyclic connectivity of cubic graphs due to Nedela and Škoviera, and second, by showing that, save for a few exceptions, a cyclically stable set in a hexagon graph of order divisible by 4 may always be chosen in such a way that it induces a tree, and its complement induces a subgraph with a single edge.

The following result is proved in [31, Theorem 17].

Proposition 3.3 (Nedela, Škoviera, 1995). The edge cyclic connectivity $\zeta(X)$ of a cubic vertex-transitive graph $X$ equals its girth $g(X)$. 
Consequently, the edge cyclic connectivity of the hexagon graph, which is an arc-transitive, and thus also a vertex-transitive cubic graph, coincides with its girth. As will soon become clear, a lower bound on the girth is needed. This is what the next proposition does, where we show that, with a few exceptions, the girth of such a graph is not less than 6 .

Proposition 3.4. Let $X$ be a cubic arc-transitive graph. Then one of the following occurs.

(i) The girth $g(X)$ of $X$ is at least 6; or

(ii) $X$ is one of the following graphs: the theta graph $\Theta_{2}, K_{4}, K_{3,3}$, the cube $Q_{3}$, the Petersen graph $G P(5,2)$ or the dodecahedron graph $G P(10,2)$.

Proof. Clearly, $\Theta_{2}$ is the only arc-transitive cubic (multi)graph of girth 2.

Let $G=$ Aut $X$. Suppose first that $g(X)=3$. Let $v \in V(X)$ and let $u_{0}, u_{1}$ and $u_{2}$ be its neighbors. By arc-transitivity there exists an automorphism $\alpha$ of $X$ fixing $v$ and cyclically permuting its neighbors, that is, $\alpha\left(u_{i}\right)=u_{i+1}, i \in \mathbb{Z}_{3}$. Since $g(X)=3$ it clearly follows that each $u_{i}$ is adjacent to the other two neighbors of $v$, and so $X \cong K_{4}$.

Suppose next that $g(X)=4$. Let $v \in V(X), N(v)=\left\{u_{i} \mid i \in \mathbb{Z}_{3}\right\}$ and $\alpha \in G_{v}$ have the same meaning as above. Since $g(X)=4$, there are no edges in $N(v)$, but there must exist, say, a vertex $x_{01}$ which is adjacent to both $u_{0}$ and $u_{1}$. If $x_{01}$ is also a neighbor of $u_{2}$, then it is easily seen that there exists a third common neighbor of $u_{0}, u_{1}$ and $u_{2}$, implying that $X \cong K_{3,3}$. If on the other hand, $x_{01}$ is not adjacent to $u_{2}$, then there must exist vertices $x_{12}$ and $x_{20}$ which are common neighbors of, respectively, $u_{1}$ and $u_{2}$, and of $u_{2}$ and $u_{0}$. But then, using the fact that $X$ is an arc-transitive graph of girth 4 , one can easily show that the three vertices $x_{01}, x_{12}$ and $x_{20}$ have a common neighbor, forcing $X$ to be the cube $Q_{3}$.

Finally, suppose that $g(X)=5$. We show first that the order of $G$ is divisible by 5 . Let $v \in V(X)$, and let $N(v)=\left\{u_{i} \mid i \in \mathbb{Z}_{3}\right\}$ be its neighborhood. We may assume that the 2-arc $u_{0} v u_{1}$ is contained in a 5-cycle. By arc-transitivity the arc $v u_{2}$ must also be contained in a 5-cycle, and so either the 2-arc $u_{0} v u_{2}$ or the 2-arc $u_{1} v u_{2}$ is contained in a 5 -cycle. But then both 2-arcs are on a 5-cycle (in view of the fact that the vertex stabilizer $G_{v}$ contains an element of order 3 which cyclically permutes the neighbors $u_{i}, i \in \mathbb{Z}_{3}$ ). In short, an edge of $X$ is contained in at least two distinct 5-cycles. On the other hand, an edge of $X$ cannot be contained in more than four 5-cycles. Namely, since $g(X)=5$, a 3 -arc of $X$ is contained in at most one 5-cycle, and so a 2 -arc of $X$ is contained in at most two 5-cycles, and a 1-arc in at most four 5-cycles. More precisely, either each 3-arc in $X$ gives rise to a unique 5-cycle or each 2 -arc gives rise to a unique 5-cycle. In the first case there is a total of $6 n / 5$ cycles of length 5 in $X$ (with each edge on four 5-cycles), and in the second case there is a total of $3 n / 5$ cycles of length 5 in $X$ (with each edge on two 5 -cycles), where $n$ is the order of $X$. In both cases we see that 5 divides $n$. In particular, $X$ has an automorphism of order 5 .

Next, by considering the action of $G$ on the set $\mathcal{C}$ of all 5-cycles in $X$, we show that this automorphism of $X$ of order 5 may be chosen in such a way that it rotates a 5-cycle in $X$ (and so in the terminology of [11], $X$ has a consistent 5-cycle). This will prove crucial in the final steps of our proof. To do that it suffices to show that there exists an 
automorphism of order 5 fixing a 5-cycle. Note that, by the classical result of Tutte [34], we have $|G|=3 \cdot 2^{r} \cdot n$, where $r \leq 2$, as $G$ is at most 3 -arc-transitive. Let $P$ be a Sylow 5 -subgroup of $G$, say of order $5^{k}, k \geq 1$. In view of the above remarks on the cardinality of $\mathcal{C}$, we can easily see that either $G$ is transitive on $\mathcal{C}$ or $G$ has two equal length orbits in its action on $\mathcal{C}$. But the important thing is that $|\mathcal{C}|$ is of the form $5^{k-1} \cdot m$, where $(5, m)=1$, and so there must be an orbit in the action of $P$ on $\mathcal{C}$ of cardinality $5^{j}$, where $j<k$. Consequently, a stabilizer in the action of $G$ on $\mathcal{C}$ contains a nonidentity element of $P$, and so, in particular, an element of order 5 of $P$. Therefore there exists a 5 -cycle $C=v_{0} v_{1} v_{2} v_{3} v_{4}$ in $X$ and an automorphism of order 5 of $X$, call it $\rho$, fixing $C$. But since $X$ is a cubic graph, $\rho$ must necessarily be fixed-point-free in its action on $V(X)$, and hence semiregular with all orbits of length 5 . It follows that $\rho$ cyclically rotates $C$, that is, with no loss of generality, $\rho\left(v_{i}\right)=v_{i+1}$ for each $i \in \mathbb{Z}_{5}$.

Let $u_{i}, i \in \mathbb{Z}_{5}$, respectively, be the additional neighbors of $v_{i}, i \in \mathbb{Z}_{5}$, so that $\rho\left(u_{i}\right)=$ $u_{i+1}$ for all $i \in \mathbb{Z}_{5}$. Supposing first that $U=\left\{u_{i} \mid i \in \mathbb{Z}_{5}\right\}$ induces a cycle, one may easily deduce that $X$ is isomorphic to the Petersen graph $G P(5,2)$. We may therefore assume that $U$ is an independent set of vertices. Now if the additional neighbors of $u_{i}, i \in \mathbb{Z}_{5}$, were in two orbits of $\rho$, then no edge $v_{i} u_{i}$ would be contained in a 5 -cycle. Hence there is a third orbit $W=\left\{w_{i} \mid i \in \mathbb{Z}_{5}\right\}$ of $\rho$, with $\rho\left(w_{i}\right)=w_{i+1}$ for all $i \in \mathbb{Z}_{5}$, containing all of the additional neighbors of vertices in $U$. Of course, $W$ is an independent set of vertices, and there is a fourth orbit $Q=\left\{q_{i} \mid i \in \mathbb{Z}_{5}\right\}$ of $\rho$ containing the additional neighbors of vertices in $W$. But now in order for the edges with one endvertex in $W$ and the other endvertex in $Q$ to lie on a 5-cycle, the orbit $Q$ must necessarily induce a cycle. In other words, $\rho$ has precisely four orbits and so $X$ is a nonbipartite cubic arc-transitive graph of order 20. Hence $X$ is isomorphic to the graph of the dodecahedron, that is, the graph $G P(10,2)$ in the generalized Petersen graph notation.

Using the previous two results we will give a refinement of the Payan and Sakarovitch result in Proposition 3.2 by showing that for cubic arc-transitive graphs of girth 6 the maximum cyclically stable subset may always be chosen to induce a tree.

Proposition 3.5. Let $X$ be a cubic arc-transitive graph of order $n \equiv 0(\bmod 4)$, not isomorphic to any of the following graphs: $K_{4}$, the cube $Q_{3}$, or the dodecahedron graph $G P(10,2)$. Then there exists a cyclically stable subset $S$ of $V(X)$ which induces a tree and such that $V(X) \backslash S$ induces a graph with a single edge.

Proof. Observe that, in view of Proposition 3.4, the girth of $X$ is at least 6, and hence, in view of Proposition 3.3. $X$ is a cyclically 6-edge-connected graph. Note also that the statement of this proposition really says that in part (ii) of Proposition 3.2. a particular one of the two possibilities may be chosen.

We proceed as follows. Let us first modify our graph $X$ by deleting a pair of adjacent vertices, say $u$ and $v$. This modified graph $Y=X-\{u, v\}$ has $n-2$ vertices with the two neighbors $u_{1}$ and $u_{2}$ of $u$ and the two neighbors $v_{1}$ and $v_{2}$ of $v$ having valency 2, and all the remaining vertices having valency 3 . "Forgetting" the four vertices $u_{1}, u_{2}, v_{1}$ and $v_{2}$, we may therefore also think of this modified graph as being cubic of order $n-6$. The important thing however is that $Y$ must be a cyclically 4-edge-connected graph. Namely, 
taking two vertex-disjoint cycles $C_{1}$ and $C_{2}$ in $Y$, a maximum number of additional paths separating these two cycles one can obtain by adding the vertices $u$ and $v$ (and all their neighbors) is 2. This would occur if each of the two vertices $u$ and $v$ had one of their two (additional) neighbors in $C_{1}$ and the other in $C_{2}$. We conclude that by going from $Y$ back to the original graph $X$, the edge cyclic connectivity can go up by at most 2 . Since $X$ is cyclically 6-edge-connected it follows that $Y$ is cyclically 4-edge-connected.

But the order of $Y$ is congruent to 2 modulo 4 and by part (i) of Proposition 3.2 (in this particular instance we are forgetting the four neighbors to make $Y$ cubic!), there exists a maximum cyclically stable subset $R$ of $V(Y)$ inducing a tree and such that its complement $V(Y) \backslash R$ is an independent set of vertices. Now the maximum cyclically stable subset $S$ of $V(X)$ is obtained by taking $S=R \cup\left\{u_{1}, u_{2}, v_{1}, v_{2}\right\}$. (The edge $u v$ is thus the single edge of the graph induced on the complement $V(X) \backslash S$.)

\section{Proof Theorem 1.1}

Suppose first that $|G| \equiv 2(\bmod 4)$. Then by Proposition 2.1, we have $s \equiv 2(\bmod 4)$. As demonstrated in Section 2 a tree in $\operatorname{Hex}(X)$ whose complement is an independent set of vertices gives rise to a Hamilton tree of faces in the Cayley map associated with $X$ and thus to a Hamilton cycle in $X$. In view of Example 2.2. which takes care of the case $\operatorname{Hex}(X) \cong \Theta_{2}$, and Propositions 3.2 3.4 which combined together take care of the case $\operatorname{Hex}(X) \approx \Theta_{2}$, the graph $X$ is then clearly hamiltonian. As for the case when $|G| \equiv 0(\bmod 4)$, we use Examples 2.4, 2.6 and 2.7 for the case when $\operatorname{Hex}(X)$ is isomorphic, respectively, to one of $K_{4}, Q_{3}$ or $G P(10,2)$ and Proposition 3.5 for the case when $\operatorname{Hex}(X) ¥ K_{4}, Q_{3}, G P(10,2)$, to ensure the existence in the hexagon graph $\operatorname{Hex}(X)$ of a tree whose vertex complement (in $\operatorname{Hex}(X))$ is a graph with a single edge. This tree then translates in $X$ into a tree of faces in the Cayley map (associated with $X$ ) whose boundary misses only two adjacent vertices, namely, those two adjacent vertices in $X$ with the corresponding edge being shared by the two hexagons which (in $\operatorname{Hex}(X)$ ) are the endvertices of the single edge in the complement of the tree chosen above. Consequently, $X$ contains a long cycle missing only two adjacent vertices. In particular, $X$ has a Hamilton path in this case. This completes the proof of Theorem 1.1 .

Acknowledgments. The authors wish to thank Yuqing Chen, Marston Conder, Gorazd Lah, Caiheng Li, Klavdija Kutnar, Denis Sjerve, Ron Solomon, and Tsuyi Yang for helpful conversations about the material in this article.

Research of D. Marušič was supported in part by "Agencija za raziskovalno dejavnost Republike Slovenije", research program P1-0285.

\section{References}

[1] Alspach, B.: Hamiltonian cycles in vertex-transitive graphs of order $2 p$. In: Proc. Tenth Southeastern Conference on Combinatorics, Graph Theory and Computing (Boca Raton, FL, 1979), Congr. Numer. XXIII-XX, Utilitas Math., Winnipeg, 131-139 (1979) Zbl 0425.05035 MR 0561039 
[2] Alspach, B.: The classification of hamiltonian generalized Petersen graphs. J. Combin. Theory Ser. B 34, 293-312 (1983) Zbl 0516.05034 MR 0714452

[3] Alspach, B.: Lifting Hamilton cycles of quotient graphs. Discrete Math. 78, 25-36 (1989) Zbl 0702.05054 MR 1020643

[4] Alspach, B.: Hamilton cycles in metacirculant graphs with prime power cardinal blocks. In: Graph Theory in Memory of G. A. Dirac (Sandbjerg, 1985), Ann. Discrete Math. 41, NorthHolland, Amsterdam, 7-16 (1989) Zbl 0665.05024 MR 0975986

[5] Alspach, B., Chen, C. C., McAvaney, K.: On a class of Hamiltonian laceable 3-regular graphs. Discrete Math. 151, 19-38 (1996) Zbl 0855.05078 MR 1391247

[6] Alspach, B., Durnberger, E., Parsons, B.: Hamilton cycles in metacirculant graphs with prime cardinality blocks. In: Cycles in Graphs (Burnaby, 1982), Ann. Discrete Math. 27, NorthHolland, Amsterdam, 27-34 (1985) Zbl 0588.05027| MR 0821504

[7] Alspach, B., Locke, S., Witte, D.: The Hamilton spaces of Cayley graphs on abelian groups. Discrete Math. 82, 113-126 (1990) Zbl 0699.05036 MR 1057481

[8] Alspach, B., Parsons, B.: On Hamiltonian cycles in metacirculant graphs. In: Algebraic and Geometric Combinatorics, Ann. Discrete Math. 15, North-Holland, Amsterdam, 1-7 (1982) Zbl 0493.05042 MR 0772579

[9] Alspach, B., Qin, Y. S.: Hamilton-connected Cayley graphs on Hamiltonian groups. Europ. J. Combin. 22, 777-787 (2001) Zbl 0983.05050 MR 1848324

[10] Alspach, B., Zhang, C. Q.: Hamilton cycles in cubic Cayley graphs on dihedral groups. Ars Combin. 28, 101-108 (1989) Zbl 0722.05047 MR 1039136

[11] Biggs, N. L.: Aspects of symmetry in graphs. In: Algebraic Methods in Graph Theory, Vol. I, II (Szeged, 1978), Colloq. Math. Soc. János Bolyai 25, North-Holland, 27-35 (1981) Zbl 0485.05030 MR 0642030

[12] Chen, Y. Q.: On Hamiltonicity of vertex-transitive graphs and digraphs of order $p^{4}$. J. Combin. Theory Ser. B 72, 110-121 (1998) Zbl 0894.05038 MR 1604701

[13] Cherkassof, M., Sjerve, D.: On groups generated by three involutions, two of which commute. In: The Hilton Symposium (Montreal, 1993), CRM Proc. Lecture Notes 6, Amer. Math. Soc., Providence, 169-185 (1994) Zbl 0818.20035

[14] Conway, J., Sloane, N., Wilks, A.: Gray codes for reflection groups. Graphs Combin. 5, 315325 (1989) Zbl 0684.20036 MR 1032382

[15] Curran, S., Gallian, J. A.: Hamiltonian cycles and paths in Cayley graphs and digraphs-a survey. Discrete Math. 156, 1-18 (1996) Zbl 0857.05067 MR 1405010

[16] Dobson, E., Gavlas, H., Morris, J., Witte, D.: Automorphism groups with cyclic commutator subgroup and Hamilton cycles. Discrete Math. 189, 69-78 (1998) Zbl 0957.05054 MR 1637709

[17] Durnberger, E.: Connected Cayley graphs of semi-direct products of cyclic groups of prime order by abelian groups are hamiltonian. Discrete Math. 46, 55-68 (1983) Zbl 0521.05046 MR 0708162

[18] Glover, H. H., Yang, T. Y.: A Hamilton cycle in the Cayley graph of the (2, $p, 3)$-presentation of $\mathrm{PSl}_{2}(p)$. Discrete Math. 160, 149-163 (1996) Zbl 0864.05061 MR 1417567

[19] Gould, R. J., Roth, R. L.: A recursive algorithm for Hamiltonian cycles in the $(1, j, n)$-Cayley graph of the alternating group. In: Graph Theory with Applications to Algorithms and Computer Science (Kalamazoo, ML, 1984), Wiley, New York, 351-369 (1985) Zbl 0578.05048 MR 0812676

[20] Jaeger, F.: On vertex-induced forests in cubic graphs. In: Proc. Fifth Southeastern Conference on Combinatorics, Graph Theory and Computing (Boca Raton, FL, 1974), Congr. Numer. X, Utilitas Math., Winnipeg, 501-512 (1974) Zbl 0307.05102 MR 0357182 
[21] Keating, K., Witte, D.: On Hamilton cycles in Cayley graphs in groups with cyclic commutator subgroup. In: Cycles in Graphs (Burnaby, 1982), Ann. Discrete Math. 27, North-Holland, Amsterdam, 89-102 (1985) Zbl 0573.05029 MR 0821508

[22] Lovász, L.: Problem 11. In: Combinatorial Structures and Their Applications (Calgary, 1969), Gordon and Breach, New York, 243-246 (1970)

[23] Lübeck, F., Malle, G.: (2, 3)-generation of exceptional groups. J. London Math. Soc. 59, 109122 (1999) Zbl 0935.20021 MR 1688493

[24] Malle, G., Saxl, J., Weigel, T.: Generation of classical groups, Geom. Dedicata 49, 85-116 (1994) Zbl 0832.20029 MR 1261575

[25] Marušič, D.: Hamilonian circuits in Cayley graphs. Discrete Math. 46, 49-54 (1983) Zbl 0515.05042 MR 0708161

[26] Marušič, D.: Vertex transitive graphs and digraphs of order $p^{k}$. In: Cycles in Graphs (Burnaby, 1982), Ann. Discrete Math. 27, North-Holland, Amsterdam, 115-128 (1985) Zbl 0571.05024 MR 0821510

[27] Marušič, D.: Hamiltonian cycles in vertex symmetric graphs of order $2 p^{2}$. Discrete Math. 66, 169-174 (1987) Zbl 0626.05037 MR 0900940

[28] Marušič, D.: On vertex-transitive graphs of order $q p$. J. Combin. Math. Combin. Comput. 4, 97-114 (1988) Zbl 0666.05040 MR 0978524

[29] Marušič, D., Parsons, T. D.: Hamiltonian paths in vertex-symmetric graphs of order $5 p$. Discrete Math. 42, 227-242 (1982) Zbl 0501.05041 MR 0677056

[30] Marušič, D., Parsons, T. D.: Hamiltonian paths in vertex-symmetric graphs of order $4 p$. Discrete Math. 43, 91-96 (1983) Zbl 0514.05037 MR 0680307

[31] Nedela, R., Škoviera, M.: Atoms of cyclic connectivity in cubic graphs. Math. Slovaca 45, 481-499 (1995) Zbl 0844.05066 MR 1390702

[32] Payan, C., Sakarovitch, M.: Ensembles cycliquement stables et graphes cubiques. Cahiers Centre Études Recherche Opér. 17, 319-343 (1975) Zbl 0314.05101 MR 0416965

[33] Stein, A.: 1 $\frac{1}{2}$-generation of simple groups. Beiträge Algebra Geom. 39, 349-358 (1998) Zbl 0924.20027 MR 1642676

[34] Tutte, W. T.: A family of cubical graphs. Proc. Cambridge Philos. Soc. 43, 459-474 (1947) Zbl 0029.42401 MR 0021678

[35] Witte, D.: On Hamiltonian circuits in Cayley diagrams. Discrete Math. 38, 99-108 (1982) Zbl 0474.05036 MR 0676525

[36] Witte, D.: On Hamilton cycles in Cayley graphs in groups with cyclic commutator subgroup. Discrete Math. 27, 89-102 (1985) Zbl 0573.05029 MR 0821508

[37] Witte, D.: Cayley digraphs of prime-power order are Hamiltonian. J. Combin. Theory Ser. B 40, 107-112 (1986) Zbl 0558.05024 MR 0830597

[38] Woldar, A.: On Hurwitz generation and genus actions of sporadic groups. Illinois J. Math. 33, 416-437 (1989) Zbl 0654.20014 MR 0996351 